Tobias Jahnke • Wilhelm Huisinga ${ }^{\star}$

\title{
Solving the chemical master equation for monomolecular reaction systems analytically
}

the date of receipt and acceptance should be inserted later

\begin{abstract}
The stochastic dynamics of a well-stirred mixture of molecular species interacting through different biochemical reactions can be accurately modelled by the chemical master equation (CME). Research in the biology and scientific computing community has concentrated mostly on the development of numerical techniques to approximate the solution of the CME via many realizations of the associated Markov jump process. The domain of exact and/or efficient methods for directly solving the CME is still widely open, which is due to its large dimension that grows exponentially with the number of molecular species involved. In this article, we present an exact solution formula of the CME for arbitrary initial conditions in the case where the underlying system is governed by monomolecular reactions. The solution can be expressed in terms of the convolution of multinomial and product Poisson distributions with time-dependent parameters evolving according to the traditional reaction-rate equations. This very structured representation allows to deduce any property of the solution. The model class includes many interesting examples and may also be used as the starting point for the design of new numerical methods for the CME of more complex reaction systems.
\end{abstract}

Keywords Chemical master equation - explicit solution formula . continuous-time Markov process · convergence to steady state

Mathematics Subject Classification (2000) 92C45 - 60J25 - 34A05

* Supported within the DFG Research Center Matheon "Mathematics for key technologies", Berlin.

T. Jahnke and W. Huisinga, Freie Universität Berlin, Fachbereich Mathematik und Informatik, Arnimallee 2-6, 14195 Berlin.

E-mail: \{jahnke,huisinga\}@math.fu-berlin.de. 


\section{Introduction}

The growing interest in stochastic models for biochemical reaction systems has lead to increasing activities in the design of efficient numerical methods for the solution of the underlying chemical master equation (CME) $[10,12$, $3,18,1,15,4,19]$. Almost all current approaches indirectly approximate the CME by generating a statistically large number of realizations of the associated continuous-time, discrete state space Markov jump process. The domain of numerical or analytical techniques for directly solving the CME is still widely open, which is mainly due to the fact that the dimension scales exponentially with the number of molecular species involved. The CME can be understood as a huge system of coupled ordinary differential equations: There is one differential equation per state of the system, in contrast to the traditional reaction-rate approach where only one differential equation per species is required. For example, a rather small system of only three species with molecule numbers varying between, say, 0 and 100 , contains $100^{3}$ different states, and 1000000 coupled differential equations have to be solved in order to determine the probability density of the CME! As a consequence, solving the CME with standard numerical integrators is usually cumbersome and often even impossible.

Analytical solutions of the CME are only known for special reaction systems with particular initial conditions; these include, e.g., closed (mass conserving) linear reaction systems with multinomial initial distributions $[6$, 8], open linear reaction systems with Poisson initial distribution [9], or a two-component, linear open system with deterministic initial conditions $[20$, Chap. 8.4]. Recently, Gadgil et al. [8] presented differential equations for the mean and the variance of a general system of first-order reactions, but did not derive an analytical solution formula for the probability distribution. Concerning the limit behavior of the CME, it is known that the equilibrium distribution of a finite closed system of linear reactions is multinomial, while the equilibrium distribution of an open system of linear reactions is Poisson in each component (see, e.g., [8] and references therein), but to the best of our knowledge, nothing is known about the general time-dependent transient behavior for arbitrary initial conditions, including the important class of deterministic initial conditions.

In this article we consider chemical reaction systems with arbitrarily many species and states, but assume that only monomolecular reactions occur. Under this condition we derive an explicit formula for the exact solution of the CME. For the generic case of deterministic initial conditions, the solution turns out to be the convolution of multinomial and product Poisson distributions with time-dependent parameters evolving according to the traditional reaction-rate equations. All previously derived results on monomolecular reaction systems can be restated in terms of our representation. Although the vast majority of biochemical systems includes bimolecular or more complex reactions, many interesting applications belong to the class of monomolecular reaction systems: conformational dynamics of proteins and RNA, simple 
birth and death processes, bacteriophage dynamics, queing systems, migration processes, gating of ion chanels, hydrolysis etc. (see, e.g., [8,7] and reference therein).

Moreover, we believe that studying monomolecular reactions is worthwhile for additional reasons: (1) Recent developments in speeding up the stochastic simulation algorithm by treating isolated subsystems (the so-called virtual fast process) analytically require the solution of the CME for these subsystems [5]. The virtual fast process will often be related to systems of monomolecular reactions (with rates depending on slow chemical species), although the overall system involves bimolecular reactions. (2) The performance of new numerical methods has to be evaluated, typically by applying the new method to a problem with known solution. Here, non-trivial, time-dependent solutions of chemical reactions systems are required. (3) The monomolecular case can be seen as a starting point for the construction of new numerical schemes. Many numerical methods are devised in such a way that they yield the exact solution in special cases, like monomolecular reaction systems for the CME. Representing the solution as a linear combination of suitably chosen basis functions and exploiting linearity of the equation, such a representation would, at the cost of a hopefully small error, reduce the number of degrees of freedom considerably. Work in this direction is currently in progress.

Outline: Section 2 sets the scene and gives a short summary of the deterministic and the stochastic approach to reaction kinetics. In Section 3 explicit solution formulas for the monomolecular CME under specific initial conditions are presented. For the convenience of the reader, we recapitulate the known result that the solution of the CME for closed systems and multinomial initial conditions stays multinomial [8]. Then, a corresponding proposition for the Poisson distribution and open systems is presented. In our main theorem, both results are combined and extended in such a way that for arbitrary initial conditions an explicit solution formula of the monomolecular CME is obtained. In Section 4 we discuss properties of the solution distribution, like expectation, variance, convergence to steady states and marginal distributions. Our results are illustrated in Section 5 in application to some simple examples previously discussed in the literature. The last section sketches possible extensions and provides an outlook.

\section{Stochastic and deterministic reaction kinetics}

We study a reaction system with $n \in \mathbb{N}$ different species or substances $S_{1}, \ldots, S_{n}$ subject to the following reaction channels:

$$
\begin{array}{llll}
\text { Reaction } R_{j k}: & S_{j} \stackrel{c_{j k}}{\longrightarrow} S_{k} & \text { conversion }(j \neq k) \\
\text { Reaction } R_{0 k}: & \star \underset{c_{0 k}}{\longrightarrow} S_{k} & \text { production from source or inflow } \\
\text { Reaction } R_{j 0}: & S_{j} \stackrel{c_{j 0}}{ } \star & \text { degradation or outflow }
\end{array}
$$

These reactions are referred to as monomolecular reactions. In comparison to general first-order reactions, we do not include (auto)catalytic reactions 
$S_{j} \stackrel{c}{\longrightarrow} S_{j}+S_{k}$, nor splitting reactions $S_{j} \stackrel{c}{\longrightarrow} S_{l}+S_{k}$ with $j \neq l, k$. For possible generalisations, see Section 6 .

The rates $c_{j k}$ are assumed to be nonnegative and can (but need not) be time dependent, i.e., $c_{j k}=c_{j k}(t)$. For simplicity of notation, let $c_{k k} \equiv 0$ for all $k \in \mathbb{N}$ in order to exclude the trivial reaction $S_{k} \stackrel{c_{k k}}{\longrightarrow} S_{k}$. We suppose that all molecules are contained in a constant volume at constant temperature, and that the system is "well-mixed" in the sense that the molecules are uniformly distributed in space as explained in [11].

\subsection{Deterministic reaction kinetics}

In the traditional approach the concentration $C_{k}(t)$ of the substance $S_{k}$ is the solution of the reaction-rate equation

$$
\dot{C}_{k}(t)=c_{0 k}(t)+\sum_{j=1}^{n} c_{j k}(t) C_{j}(t)-\sum_{j=0}^{n} c_{k j}(t) C_{k}(t) .
$$

Hence, the vector $C(t)=\left(C_{1}(t), \ldots, C_{n}(t)\right)^{T}$ of all concentrations evolves according to

$$
\dot{C}(t)=A(t) C(t)+b(t)
$$

where $b(t) \in \mathbb{R}^{n}$ is the vector

$$
b(t)=\left(c_{01}(t), c_{02}(t), \ldots, c_{0 n}(t)\right)^{T} .
$$

and $A(t)=\left(a_{j k}(t)\right)_{j, k} \in \mathbb{N}^{n \times n}$ denotes the matrix with entries $a_{j k}(t)$ defined by

$$
a_{j k}(t)=c_{k j}(t) \quad \text { for } j \neq k \geq 1, \quad a_{k k}(t)=-\sum_{j=0}^{n} c_{k j}(t) .
$$

The definition of $A$ implies that the sum of each column is nonpositive, because

$$
\sum_{j=1}^{n} a_{j k}(t)=-c_{k 0}(t) \leq 0
$$

A matrix with property (6) and nonnegative off-diagonal elements is called a compartmental matrix [2]. Compartmental matrices have many nice features (see Proposition 4), for example the fact that the solution $C(t)$ has nonnegative entries if the initial vector $C(0)$ has nonnegative entries. This can be readily seen from (2), because $C_{k}(t)=0$ implies $\dot{C}_{k}(t) \geq 0$. Moreover, mass balance shows that $\sum_{k=1}^{n} C_{k}(t) \leq \sum_{k=1}^{n} C_{k}(0)$ for each $t=0$, and " $\leq$ " can be replaced by " $=$ " if $c_{k 0}=0$ for all $k$. 
2.2 Stochastic reaction kinetics

At any time $t$ the state of the system is described by the number of molecules of each species. Since no positional information is available the state of the system is to be understood as a random variable $X(t)=\left(X_{1}(t), \ldots, X_{n}(t)\right)$ where $X_{k}(t)$ is the number of molecules of the $k$-th species at time $t$.

The reactions are entirely defined by the stoichiometric vectors and the propensities. The stoichiometric vector $\nu^{(j k)} \in \mathbb{N}^{n}$ corresponds to state change that occurs whenever the reaction channel $R_{j k}$ fires. In case of the reaction system (1) we have

$$
\nu^{(j k)}=\varepsilon_{k}-\varepsilon_{j}, \quad \nu^{(0 k)}=\varepsilon_{k}, \quad \nu^{(j 0)}=-\varepsilon_{j}
$$

where $\varepsilon_{k}$ denotes the $k$-th column of the identity matrix in $\mathbb{R}^{n \times n}$. The propensity $\alpha_{j k}(t, x)$ is related to the reaction probability in the following way: If the system is in state $X(t)=x \in \mathbb{N}^{n}$ at time $t$, then $\alpha_{j k}(t, x) d t$ is the probability that in the next "infinitesimal" time interval $[t, t+d t]$ the reaction channel $R_{j k}$ will fire. According to [11] the propensities are given by

$$
\alpha_{j k}(t, x)= \begin{cases}c_{j k}(t) x_{j} & \text { for reaction } R_{j k} \\ c_{0 k}(t) & \text { for reaction } R_{0 k} \\ c_{j 0}(t) x_{j} & \text { for reaction } R_{j 0}\end{cases}
$$

for all $x \in \mathbb{N}^{n}$. For ease of notation we also define $\alpha_{j k}(t, x)=0$ if $x \notin \mathbb{N}^{n}$.

In this article, we determine the probability distribution

$$
P(t, x)=\mathbb{P}\left(X_{1}(t)=x_{1}, \ldots, X_{n}(t)=x_{n}\right)
$$

of the random variable $X(t)$. For convenience, we define $P(t, x)=0$ for all $x \notin \mathbb{N}^{n}$. The distribution $P$ is the solution of the CME

$$
\partial_{t} P(t, x)=\sum_{j=0}^{n} \sum_{k=0}^{n}\left(\alpha_{j k}\left(t, x-\nu^{j k}\right) P\left(t, x-\nu^{j k}\right)-\alpha_{j k}(t, x) P(t, x)\right),
$$

which can be rewritten as

$$
\begin{aligned}
\partial_{t} P(t, x)= & \sum_{k=1}^{n} c_{0 k}(t)\left(P\left(t, x-\varepsilon_{k}\right)-P(t, x)\right) \\
& +\sum_{k=1}^{n} c_{k 0}(t)\left(\left(x_{k}+1\right) P\left(t, x+\varepsilon_{k}\right)-x_{k} P(t, x)\right) \\
& +\sum_{j=1}^{n} \sum_{k=1}^{n} c_{j k}(t)\left(\left(x_{j}+1\right) P\left(t, x+\varepsilon_{j}-\varepsilon_{k}\right)-x_{j} P(t, x)\right) .
\end{aligned}
$$

The first sum of the CME (8) corresponds to the inflow reactions $R_{0 k}$, the second one to the degradation $R_{k 0}$, and the double sum represents the conversions $R_{j k}$. 
We suppose that the molecule numbers at time $t=0$ are specified in terms of some arbitrary initial probability distribution $\mu$, i.e.

$$
\mathbb{P}(X(0)=x)=\mu(x)
$$

for every $x \in \mathbb{N}^{n}$. Exploiting linearity of the CME, the problem of solving the CME for arbitrary initial distribution can be reduced to the problem of solving the CME for deterministic initial conditions, i.e.,

$$
P(0, x)=\delta_{\xi}(x)= \begin{cases}1 & \text { if } x=\xi \\ 0 & \text { otherwise }\end{cases}
$$

with $\delta_{\xi}(x)$ denoting the Kronecker symbol. Hence, our aim is to find a formula for the exact solution of (8) and (9).

\section{Explicit solution formulas for the monomolecular master equation}

In order to introduce our notation, we briefly list some basic definitions. The convolution $P_{1} \star P_{2}$ of two probability distributions $P_{1}$ and $P_{2}$ on the state space $\mathbb{N}^{n}$ is defined by

$$
\left(P_{1} \star P_{2}\right)(x)=\sum_{z} P_{1}(z) P_{2}(x-z)=\sum_{z} P_{1}(x-z) P_{2}(z)
$$

where the sum is taken over all $z \in \mathbb{N}^{n}$ such that $(x-z) \in \mathbb{N}^{n}$.

Let $|x|:=\sum_{k=1}^{n}\left|x_{k}\right|$ be the 1-norm of the vector $x$. For any $x \in \mathbb{N}^{n}$ and any $p=\left(p_{1}, \ldots, p_{n}\right) \in[0,1]^{n}$ with $|p| \leq 1$, the multinomial (or polynomial) distribution $\mathcal{M}(x, N, p)$ is given by

$$
\mathcal{M}(x, N, p)=\left\{\begin{array}{cl}
N ! \frac{(1-|p|)^{N-|x|}}{(N-|x|) !} \prod_{k=1}^{n} \frac{p_{k}^{x_{k}}}{x_{k} !} & \text { if }|x| \leq N \text { and } x \in \mathbb{N}^{n} \\
0 & \text { else. }
\end{array}\right.
$$

Finally, let $\mathcal{P}$ denote the product Poisson distribution

$$
\mathcal{P}(x, \lambda)=\frac{\lambda_{1}^{x_{1}}}{x_{1} !} \cdot \ldots \cdot \frac{\lambda_{n}^{x_{n}}}{x_{n} !} \cdot e^{-|\lambda|}, \quad x \in \mathbb{N}^{n}
$$

with parameter vector $\lambda=\left(\lambda_{1}, \ldots, \lambda_{n}\right) \in \mathbb{R}^{n}$ containing nonnegative entries, and for ease of notation put $\mathcal{P}(x, \lambda)=0$ if $x \notin \mathbb{N}^{n}$. 
3.1 Closed systems with multinomial initial distribution

In this section we assume that the system is closed ${ }^{1}$ in the sense that all inflow reactions $R_{0 k}: * \longrightarrow X_{k}$ are excluded by letting $c_{0 k}=0$ for all $k$. Moreover, suppose that instead of the deterministic initial data $(9), P(0, x)$ is a multinomial distribution. Then, this is one of the few cases where the CME (8) can be solved analytically, cf. [8].

Proposition 1 Consider the monomolecular reaction system (1) and suppose that $c_{0 k}=0$ for all $k$. If the initial distribution is the multinomial distribution

$$
P(0, x)=\mathcal{M}\left(x, N, p_{0}\right)
$$

for some $p_{0} \in[0,1]^{n}$, then the probability distribution at time $t>0$ is still a multinomial distribution

$$
P(t, x)=\mathcal{M}(x, N, p(t)) .
$$

with a parameter vector $p(t)=\left(p_{1}(t), \ldots, p_{n}(t)\right)^{T}$ evolving according to the differential equation

$$
\begin{aligned}
& \dot{p}(t)=A(t) p(t) \\
& p(0)=p_{0}
\end{aligned}
$$

with the matrix $A$ defined in (5).

Remark: The differential equation (10) is the familiar deterministic rate equation $(3)$ in the inflow-free case $b(t) \equiv(0, \ldots, 0)^{T}$. If all rates $c_{j k}$ are constant the solution of $(10)$ is simply $p(t)=\exp (t A) p_{0}$. If some of the rates are time-dependent, the matrix exponential $\exp (A(t))$ does generally not provide the solution, but the differential equation (10) can be solved numerically by using, e.g., a Magnus method [16].

Proof: A proof can be found in [8] (for constant rates), but we prove the result in a different way by verifying that $\mathcal{M}(x, N, p(t))$ is indeed the solution of the CME (8). The initial condition is obviously met. Let $x \in \mathbb{N}^{n}$ be fixed, put $y=N-|x|$ and define $q(t)=1-|p(t)|$. We first consider the case that $x_{k} \neq 0$ and $y \neq 0$. Taking the derivative of the probability distribution gives

$$
\begin{aligned}
& \frac{d}{d t} \mathcal{M}(x, N, p(t))=N ! \frac{d}{d t}\left(\frac{q^{y}(t)}{y !} \prod_{k=1}^{n} \frac{p_{k}^{x_{k}}(t)}{x_{k} !}\right) \\
& =N ! \frac{q^{y-1}(t)}{(y-1) !} \dot{q}(t) \cdot \prod_{k=1}^{n} \frac{p_{k}^{x_{k}}(t)}{x_{k} !}+N ! \frac{q^{y}(t)}{y !} \sum_{j=1}^{n} \frac{p_{j}^{x_{j}-1}(t)}{\left(x_{j}-1\right) !} \dot{p}_{j}(t) \cdot \prod_{k \neq j} \frac{p_{k}^{x_{k}}(t)}{x_{k} !} .
\end{aligned}
$$

\footnotetext{
1 The fact that some molecules can "leave" the system via the reactions $R_{k 0}$ : $X_{k} \longrightarrow *$ does not contradict the closedness of the system, because one can say that the degraded molecules belong to an additional species, say, $X_{*}$. If this is done, the total number of molecules is constant. The only particular thing about $X_{*}$ is that there is no reaction from $X_{*}$ back to any other species.
} 
From (10) we obtain

$$
\dot{p}_{j}=\sum_{i=1}^{n} c_{i j} p_{i}-\left(\sum_{i=0}^{n} c_{j i}\right) p_{j}, \quad \dot{q}=-\sum_{i=1}^{n} \dot{p}_{i}=\sum_{i=1}^{n} c_{i 0} p_{i}
$$

because $p_{i}(t) \geq 0$ for all $i$ (cf. Section 2 ). This yields

$$
\begin{aligned}
\frac{d}{d t} \mathcal{M}(x, N, p)= & N ! \frac{q^{y-1}}{(y-1) !}\left(\sum_{i=1}^{n} c_{i 0} p_{i}\right) \cdot \prod_{k=1}^{n} \frac{p_{k}^{x_{k}}}{x_{k} !} \\
& +N ! \frac{q^{y}}{y !} \sum_{j=1}^{n} \frac{p_{j}^{x_{j}-1}}{\left(x_{j}-1\right) !}\left(\sum_{i=1}^{n} c_{i j} p_{i}\right) \cdot \prod_{k \neq j} \frac{p_{k}^{x_{k}}}{x_{k} !} \\
& -N ! \frac{q^{y}}{y !} \sum_{j=1}^{n} \frac{p_{j}^{x_{j}-1}}{\left(x_{j}-1\right) !}\left(\sum_{i=0}^{n} c_{j i}\right) p_{j} \cdot \prod_{k \neq j} \frac{p_{k}^{x_{k}}}{x_{k} !} \\
=N ! \frac{q^{y-1}}{(y-1) !} & \sum_{i=1}^{n} c_{i 0}\left(x_{i}+1\right) \frac{p_{i}^{x_{i}+1}}{\left(x_{i}+1\right) !} \cdot \prod_{k \neq i} \frac{p_{k}^{x_{k}}}{x_{k} !} \\
& +N ! \frac{q^{y}}{y !} \sum_{j=1}^{n} \sum_{i=1}^{n} c_{i j} \frac{p_{j}^{x_{j}-1}}{\left(x_{j}-1\right) !}\left(x_{i}+1\right) \frac{p_{i}^{x_{i}+1}}{\left(x_{i}+1\right) !} \prod_{k \neq j, k \neq i} \frac{p_{k}^{x_{k}}}{x_{k} !} \\
& -\sum_{j=1}^{n} \sum_{i=0}^{n} c_{j i} x_{j} \mathcal{M}(x, N, p) . \\
& -\sum_{j=1}^{n} \sum_{i=0}^{n} c_{j i} x_{j} \mathcal{M}(x, N, p) .
\end{aligned}
$$

Rearranging these expressions gives

$$
\begin{aligned}
\frac{d}{d t} \mathcal{M}(x, N, p)= & \sum_{i=1}^{n} c_{i 0}\left(\left(x_{i}+1\right) \mathcal{M}\left(x+\varepsilon_{i}, N, p\right)-x_{i} \mathcal{M}(x, N, p)\right) \\
& +\sum_{j=1}^{n} \sum_{i=1}^{n} c_{i j}\left(\left(x_{i}+1\right) M\left(x+\varepsilon_{i}-\varepsilon_{j}\right)-x_{i} \mathcal{M}(x, N, p)\right)
\end{aligned}
$$

which is the CME (8) in the case $c_{0 k}=0$ for all $k=1, \ldots, n$. If $y=0$ and/or $x_{k}=0$ for some $k$, then some of the terms in the above formulas vanish because $p_{k}(t)^{0} / 0 ! \equiv 1$ and $\frac{d}{d t} p_{k}(t)^{0} / 0 ! \equiv 0$, but the same calculation can be carried out mutatis mutandis.

Proposition 1 states an explicit formula for the probability distribution under the condition that there is no inflow into the system and that the initial distribution is multinomial. Both conditions, however, are very restrictive. Nevertheless, Proposition 1 will be helpful in the proof of our main result (Theorem 1). 
3.2 Open systems with product Poisson initial distribution

In a next step towards this aim, we admit inflow reactions and state a result similar to Proposition 1 for the Poisson distribution, cf. [9].

Proposition 2 Consider a system of $n$ species subject to monomolecular reactions of type $R_{j k}, R_{0 k}$, and $R_{k 0}$. Suppose that the initial distribution is a product Poisson distribution

$$
P(0, x)=\mathcal{P}\left(x, \lambda_{0}\right)
$$

with a parameter vector $\lambda_{0}$ of nonnegative entries. Then the probability distribution at time $t>0$ is still a product Poisson distribution

$$
P(t, x)=\mathcal{P}(x, \lambda(t)) .
$$

with parameter vector $\lambda(t)$ which is the solution of the reaction rate equation

$$
\begin{aligned}
& \dot{\lambda}(t)=A(t) \lambda(t)+b(t) \\
& \lambda(0)=\lambda_{0}
\end{aligned}
$$

with $A(t)$ and $b(t)$ defined in (5) and (4).

Proof: This result is derived in [9] using moment generating functions. For convenience of the reader, we give a direct proof. Let $x \in \mathbb{N}^{n}$ and, as in the proof of Proposition 1, assume first that $x_{k} \neq 0$ for all $k$. Since

$$
\frac{d}{d t} \frac{\lambda_{k}^{x_{k}}}{x_{k} !} e^{-\lambda_{k}}=-\dot{\lambda}_{k} \frac{\lambda_{k}^{x_{k}}}{x_{k} !} e^{-\lambda_{k}}+\dot{\lambda}_{k} \frac{\lambda_{k}^{x_{k}-1}}{\left(x_{k}-1\right) !} e^{-\lambda_{k}}
$$

the derivative of the Poisson distribution is

$$
\frac{d}{d t} \mathcal{P}(x, \lambda)=-\sum_{k=1}^{n} \dot{\lambda}_{k} \mathcal{P}(x, \lambda)+\sum_{j=1}^{n} \dot{\lambda}_{j} \mathcal{P}\left(x-\varepsilon_{j}, \lambda\right) .
$$

From (11) and (6) we have

$$
\begin{aligned}
\dot{\lambda}_{j} & =\sum_{i=1}^{n} a_{j i} \lambda_{i}+b_{j}=\sum_{i=1}^{n} c_{i j} \lambda_{i}-\sum_{i=0}^{n} c_{j i} \lambda_{j}+c_{0 j} \\
\sum_{k=1}^{n} \dot{\lambda}_{k} & =-\sum_{k=1}^{n} c_{k 0} \lambda_{k}+|b|
\end{aligned}
$$

which turns the first term of (12) into

$$
\begin{aligned}
-\sum_{k=1}^{n} \dot{\lambda}_{k} \mathcal{P}(x, \lambda) & =\left(\sum_{k=1}^{n} c_{k 0} \lambda_{k}-|b|\right) \mathcal{P}(x, \lambda) \\
& =\sum_{k=1}^{n} c_{k 0}\left(x_{k}+1\right) \mathcal{P}\left(x+\varepsilon_{k}, \lambda\right)-\sum_{k=1}^{n} c_{0 k} \mathcal{P}(x, \lambda) .
\end{aligned}
$$


Using the relation $\lambda_{j} \mathcal{P}\left(x-\varepsilon_{j}, \lambda\right)=x_{j} \mathcal{P}(x, \lambda)$ we find that the second term of (12) equals

$$
\begin{aligned}
& \sum_{j=1}^{n} \dot{\lambda}_{j} \mathcal{P}\left(x-\varepsilon_{j}, \lambda\right) \\
= & \sum_{j=1}^{n} \sum_{i=1}^{n} c_{i j} \lambda_{i} \mathcal{P}\left(x-\varepsilon_{j}, \lambda\right)-\sum_{j=1}^{n} \sum_{i=0}^{n} c_{j i} \lambda_{j} \mathcal{P}\left(x-\varepsilon_{j}, \lambda\right)+\sum_{j=1}^{n} c_{0 j} \mathcal{P}\left(x-\varepsilon_{j}, \lambda\right) \\
= & \sum_{j=1}^{n} \sum_{i=1}^{n} c_{i j}\left(x_{i}+1\right) \mathcal{P}\left(x+\varepsilon_{i}-\varepsilon_{j}, \lambda\right)-\sum_{j=1}^{n} \sum_{i=1}^{n} c_{j i} x_{j} \mathcal{P}(x, \lambda) \\
& \quad \sum_{j=1}^{n} c_{j 0} x_{j} \mathcal{P}(x, \lambda)+\sum_{j=1}^{n} c_{0 j} \mathcal{P}\left(x-\varepsilon_{j}, \lambda\right) .
\end{aligned}
$$

Substituting (13) and (14) into (12) yields

$$
\begin{aligned}
\frac{d}{d t} \mathcal{P}(x, \lambda)= & \sum_{j=1}^{n} c_{0 j}\left(\mathcal{P}\left(x-\varepsilon_{j}, \lambda\right)-\mathcal{P}(x, \lambda)\right) \\
& +\sum_{k=1}^{n} c_{k 0}\left(\left(x_{k}+1\right) \mathcal{P}\left(x+\varepsilon_{k}, \lambda\right)-x_{k} \mathcal{P}(x, \lambda)\right) \\
& +\sum_{j=1}^{n} \sum_{i=1}^{n} c_{i j}\left(\left(x_{i}+1\right) \mathcal{P}\left(x+\varepsilon_{i}-\varepsilon_{j}, \lambda\right)-x_{i} \mathcal{P}(x, \lambda)\right)
\end{aligned}
$$

which is exactly the CME (8).

Now suppose that $x_{k}=0$ for one or more $k$. Without loss of generality, it can be assumed, possibly after a suitable permutation of the indices, that there is an $m \in \mathbb{N}$ such that $x_{k}=0$ for all $m<k \leq n$ and $x_{k} \neq 0$ for all $1 \leq k \leq m$. Then, the formula (12) for the derivative of $\mathcal{P}(x, \lambda)$ has to be adapted such that the second sum is only taken from 1 to $m$. Accordingly, (14) remains valid if " $\sum_{j=1}^{n} \ldots$ " is replaced by " $\sum_{j=1}^{m} \ldots$ " everywhere, and since we have defined $\mathcal{P}(x, \lambda)=0$ for $x \notin \mathbb{N}^{n}$ the CME (8) is again recovered.

Remarks: 1. A different proof of Proposition 1 is obtained by regarding the source as a compartment containing $N$ molecules, applying Proposition 1 and passing to the limit $N \rightarrow \infty$. The assertion then follows from the Poisson limit theorem which states that for $N \rightarrow \infty$ a binomial distribution converges to a Poisson distribution.

2. On the other hand, Proposition 2 can be used to prove Proposition 1 by application of the following well-known result (see [17]): If $X_{1}, \ldots, X_{n}$ are independent Poisson variables with parameters $\lambda_{1}, \ldots, \lambda_{n}$, then the conditional distribution of $X(t)=\left(X_{1}, \ldots, X_{n}\right)$ given $\sum_{i=1}^{n} X_{i}=N$ is multinomial with parameters $N$ and $p_{i}=\lambda_{i} / \sum_{i=1}^{n} \lambda_{i}$. 
3.3 General systems with arbitrary initial distribution

The above propositions state that "Poisson stays Poisson" and, in closed systems, that "multinomial stays multinomial". Though these results provide solutions of the CME their practical use is limited, because in typical applications the initial data is neither a multinomial nor Poisson distribution but a deterministic condition of the form $P(0, x)=\delta_{\xi}(x)$. However, for some special parameter values the multinomial and Poisson distribution are indeed of this form, namely

$$
\begin{aligned}
& \mathcal{P}(x, \lambda)=\delta_{\mathbf{0}}(x) \quad \Longleftrightarrow \quad \lambda=\mathbf{0} \\
& \mathcal{M}(x, N, p)=\delta_{\mathbf{0}}(x) \quad \Longleftrightarrow p=\mathbf{0} \\
& \mathcal{M}(x, N, p)=\delta_{N \varepsilon_{j}}(x) \quad \Longleftrightarrow p=\varepsilon_{j}
\end{aligned}
$$

where the symbol $\mathbf{0}$ denotes the zero vector $\mathbf{0}=(0, \ldots, 0)^{T} \in \mathbb{R}^{n}$. The first two lines correspond to the situation where no molecules exist at all, i.e. $\mathbb{P}(X=\mathbf{0})=1$. The third line is the situation where all $N$ molecules belong to the same species $S_{j}$. Based on this observation we now derive an explicit formula for the solution $P(t, x)$ that holds if the initial condition is deterministic.

Theorem 1 For any $\xi \in \mathbb{N}^{n}$ the solution of the CME (8) with initial data $P(0, \cdot)=\delta_{\xi}(\cdot)$ is

$$
P(t, \cdot)=\mathcal{P}(\cdot, \lambda(t)) \star \mathcal{M}\left(\cdot, \xi_{1}, p^{(1)}(t)\right) \star \ldots \star \mathcal{M}\left(\cdot, \xi_{n}, p^{(n)}(t)\right) .
$$

The vectors $p^{(k)}(t) \in[0,1]^{n}$ and $\lambda(t) \in \mathbb{R}^{n}$ are the solutions of the reactionrate equations

$$
\begin{array}{ll}
\dot{p}^{(k)}(t)=A(t) p^{(k)}(t), & \dot{\lambda}(t)=A(t) \lambda(t)+b(t), \\
p^{(k)}(0)=\varepsilon_{k} & \lambda(0)=\mathbf{0}
\end{array}
$$

with $A(t)$ and $b(t)$ defined in (5) and (4), respectively.

Remark. If instead of a deterministic initial condition a probability distribution is given, the solution can be obtained by superposition, as already mentioned in Section 2.2.

Proof. We split the molecules in $n+1$ different groups: For $1 \leq k \leq n$, the $k$-th group contains only those $\xi_{k}$ molecules that are of type $S_{k}$ at time $t=0$, and the zeroth group comprises all molecules that do not yet exist at $t=0$ but are created via a reaction of type $R_{0 k}$. The evolution of each group of molecules is again a random variable that will be denoted by $X^{(k)}(t)$ with $k=$ $0,1, \ldots, n$. The crucial observation is that molecules belonging to different groups evolve independently. Hence, $X(t)=X^{(0)}(t)+X^{(1)}(t)+\ldots+X^{(n)}(t)$ is the sum of independent random variables, and according to a well-known theorem [14], the joint probability distribution is given by the convolution

$$
P(t, \cdot)=P^{(0)}(t, \cdot) \star P^{(1)}(t, \cdot) \star \ldots \star P^{(n)}(t, \cdot)
$$

where $P^{(k)}(t, x)$ is the probability distribution of $X^{(k)}(t)$. These distributions, however, are immediately obtained from the previous propositions. For $1 \leq$ 
$k \leq n$ the initial distribution is $P^{(k)}(0, x)=\delta_{\xi_{k}}(x)=\mathcal{M}\left(x, \xi_{k}, \varepsilon_{k}\right)$, and according to Proposition 1 we have

$$
P^{(k)}(t, x)=\mathcal{M}\left(x, \xi_{k}, p^{(k)}(t)\right)
$$

where $p^{(k)}$ is the solution of the left differential equation in (16). Since the initial distribution of the zeroth group is $P^{(0)}(0, x)=\mathcal{P}(x, \mathbf{0})=\delta_{\mathbf{0}}(x)$, Proposition 2 states that the probability distribution is

$$
P^{(0)}(t, x)=\mathcal{P}(x, \lambda(t))
$$

with $\lambda$ being the solution of the right differential equation in (16). This proves the assertion.

Our main result represents the exact solution of the CME (8) in terms of well-known probability distributions with parameters evolving according to reaction-rate equations. This very structured representation allows to deduce any property of the solution, as exemplified in the next section.

\section{Properties of the solution distribution}

\subsection{Marginal distributions}

When the exact solution of the CME (8) is to be visualized, one is restricted to low-dimensional projections. Therefore, we derive mariginal distribution formulas for the solution distribution in this subsection.

For a vector-valued random variable $X=\left(X_{1}, \ldots, X_{n}\right)^{T} \in \mathbb{N}^{n}$ and any number $j \in 1, \ldots, n-1$ we define two lower-dimensional random variables

$$
Y=\left(X_{1}, \ldots, X_{j}\right)^{T} \in \mathbb{N}^{j}, \quad Z=\left(X_{j+1}, \ldots, X_{n}\right)^{T} \in \mathbb{N}^{n-j}
$$

such that $X=(Y, Z)^{T}$. Any other partition of $X$ can be obtained by an appropriate permutation of the indices. Moreover, let

$$
P(y, z)=\mathbb{P}(Y=y, Z=z), \quad F_{Y}(y)=\mathbb{P}(Y=y), \quad F_{Z}(z)=\mathbb{P}(Z=z)
$$

be the corresponding probability distributions. Then $F_{Y}$ and $F_{Z}$ are called marginal distributions of $P$ and are given by

$$
\sum_{z \in \mathbb{N}^{n}-j} P(y, z)=F_{Y}(y), \quad \sum_{y \in \mathbb{N}^{j}} P(y, z)=F_{Z}(z),
$$

cf. [14]. We show that the marginal distribution of the solution of (8) has the same form as the distribution (15) itself, but in a lower dimension. 
Proposition 3 Let $P(t, x)$ be the solution (15) of the CME (8). Moreover, fix $j \in 1, \ldots, n-1$ and define $x=(y, z)$ with $y=\left(x_{1}, \ldots, x_{j}\right)$ and $z=$ $\left(x_{j+1}, \ldots, x_{n}\right)$. Then, the marginal distribution

$$
F_{Y}(t, y)=\sum_{z \in \mathbb{N}^{n-j}} P(t,(y, z))=\sum_{x_{j+1}} \sum_{x_{j+2}} \cdots \sum_{x_{n}} P(t, x)
$$

of $P(t, x)$ is given by

$$
\begin{array}{r}
F_{Y}(t, y)=\left(\mathcal{P}(\cdot, \tilde{\lambda}(t)) \star \mathcal{M}\left(\cdot, \xi_{1}, \tilde{p}^{(1)}(t)\right) \star \quad \ldots\right. \\
\left.\ldots \star \mathcal{M}\left(\cdot, \xi_{n}, \tilde{p}^{(n)}(t)\right)\right)(y)
\end{array}
$$

where $\tilde{p}^{(i)}=\left(p_{1}^{(i)}, \ldots, p_{j}^{(i)}\right)^{T}$ and $\tilde{\lambda}=\left(\lambda_{1}, \ldots, \lambda_{j}\right)^{T}$ only contain the first $j$ entries of the parameter vectors $p^{(i)}(t) \in[0,1]^{n}$ and $\lambda(t) \in \mathbb{R}^{n}$ defined by the differential equations (16).

Remark. Note that (17) does not depend explicitly on neither $p_{j+1}^{(i)}, \ldots, p_{n}^{(i)}$ nor $\lambda_{j+1}, \ldots, \lambda_{n}$, but these values still have to be computed because they are coupled with the other entries via the differential equation (16).

Proof. It can be shown that the marginal distributions of the multinomial and the Poisson distribution are

$$
\begin{aligned}
\sum_{z \in \mathbb{N}^{n-j}} \mathcal{M}((y, z), N, p) & =\mathcal{M}(y, N, \tilde{p}) \\
\sum_{z \in \mathbb{N}^{n-j}} \mathcal{P}((y, z), \lambda) & =\mathcal{P}(y, \tilde{\lambda})
\end{aligned}
$$

with $\tilde{p}=\left(p_{1}, \ldots, p_{j}\right)^{T}$ and $\tilde{\lambda}=\left(\lambda_{1}, \ldots, \lambda_{j}\right)^{T}$ (cf. [17]). Now, the statement follows from the fact that computing the convolution and passing to the marginal distribution commutes.

\subsection{Expectation and variance}

The expectation $\mathbb{E}(X)$ and covariance matrix $\operatorname{Cov}\left(X_{j}, X_{k}\right)=\mathbb{E}\left(X_{j}-\mathbb{E} X_{j}\right)\left(X_{k}-\right.$ $\left.\mathbb{E} X_{k}\right)$ of the probability distribution $P(t, x)$ can easily be calculated. It is known that an $n$-dimensional random variable $Y$ with multinomial distribution $\mathcal{M}(x, N, p)$ has

$$
\mathbb{E}(Y)=N p, \quad \operatorname{Cov}\left(Y_{j}, Y_{k}\right)=\left\{\begin{array}{cc}
-N p_{j} p_{k} & \text { if } j \neq k \\
N p_{k}\left(1-p_{k}\right) & \text { if } j=k
\end{array}\right.
$$

(see [17]). If $Y$ is distributed according to the product Poisson distribution $\mathcal{P}(x, \lambda)$, then

$$
\mathbb{E}(Y)=\lambda, \quad \operatorname{Cov}\left(Y_{j}, Y_{k}\right)=0 \text { if } j \neq k, \quad \operatorname{Cov}\left(Y_{k}, Y_{k}\right)=\lambda_{k} .
$$


The sum of two independent random variables $Y_{1}$ and $Y_{2}$ has expectation $\mathbb{E}\left(Y_{1}+Y_{2}\right)=\mathbb{E}\left(Y_{1}\right)+\mathbb{E}\left(Y_{2}\right)$ and covariance $\operatorname{Cov}\left(Y_{1}, Y_{2}\right)=0$ (cf. [14]). Together with the (bi-)linearity of $\mathrm{Cov}$ and $\mathbb{E}$, this yields

$$
\begin{aligned}
\mathbb{E}(X(t)) & =\lambda(t)+\sum_{k=1}^{n} \xi_{k} p^{(k)}(t) \\
\operatorname{Cov}\left(X_{j}, X_{k}\right) & =\left\{\begin{array}{cl}
\sum_{i=1}^{n} \xi_{i} p_{j}^{(i)}\left(1-p_{j}^{(i)}\right)+\lambda_{j} & \text { if } j=k \\
-\sum_{i=1}^{n} \xi_{i} p_{j}^{(i)} p_{k}^{(i)} & \text { else }
\end{array}\right.
\end{aligned}
$$

with $p^{(k)}$ and $\lambda(t)$ from (16). It is well-known that the expectation $\mathbb{E}(X)$ of the monomolecular reaction system (1) evolves according to the deterministic rate equation (3) used in "classical" reaction kinetics. This can easily be seen from the above expression: At $t=0$ we have

$$
\mathbb{E}(X(0))=\lambda(0)+\sum_{k=1}^{n} \xi_{k} p^{(k)}(0)=\sum_{k=1}^{n} \xi_{k} \varepsilon_{k}=\xi,
$$

and taking the derivative of $\mathbb{E}(X(t))$ yields via (16)

$$
\begin{aligned}
\frac{d}{d t} \mathbb{E}(X(t)) & =\dot{\lambda}(t)+\sum_{k=1}^{n} \xi_{k} \dot{p}^{(k)}(t)=A(t) \lambda(t)+b(t)+\sum_{k=1}^{n} \xi_{k} A p^{(k)}(t) \\
& =A(t) \mathbb{E}(X(t))+b(t)
\end{aligned}
$$

\subsection{Steady states and convergence}

As stated in the introduction, it is well-known that the equilibrium distribution of a finite closed system is multinomial, while it is product Poisson for open systems. In this section, we deduce much stronger results investigating the convergence of the system from an arbitrary initial distribution to a steady state.

Assume that the initial distribution is given in terms of some probability distribution $\mu$, i.e., $P(0, x)=\mu(x)$ for every $x \in \mathbb{N}^{n}$. Then, expoiting linearity of the CME, the distribution at time $t>0$ can be written as

$$
P(t, \cdot)=\sum_{\xi \in \mathbb{N}^{n}} \mu(\xi) P_{\xi}(t, \cdot)
$$

where $P_{\xi}(t, \cdot)$ satisfies the $\mathrm{CME}(8)$ with initial condition $P_{\xi}(0, \cdot)=\delta_{\xi}$. In the following we will see that the above representation (20) can be much simplified for the classes of closed and open systems, exploiting the structure of the probability distributions $P_{\xi}(t, \cdot)$ obtained in Theorem 1 . To do so, we impose two additional and natural assumptions on the reaction system:

(A1) All reaction rates $c_{j k}$ are constant. 
(A2) The matrix $A$ is irreducible which means that there is no block partition of the form

$$
A=\left(\begin{array}{cc}
A_{11} & 0 \\
A_{21} & A_{22}
\end{array}\right) \text {. }
$$

Concerning (A1), a steady state might not exist, if the rates are time-dependent, while (A2) guarantees that the system cannot be decomposed into two or more completely separated parts (see, e.g., [2]). Next, we quote some useful results about compartmental matrices.

\section{Proposition 4 (Theorems 12.1, 12.3, 13.1, and 13.2 in [2])}

- The real part of any eigenvalue $\sigma_{k}$ of $A$ is nonpositive and there are no purely imaginary eigenvalues:

$$
\operatorname{Re}\left(\sigma_{k}\right) \leq 0 \quad \text { and } \quad \operatorname{Re}\left(\sigma_{k}\right)=0 \quad \Longleftrightarrow \quad \sigma_{k}=0 .
$$

- Let $\sigma_{1}$ be the eigenvalue with the smallest absolute value, i.e. $\left|\sigma_{1}\right| \leq\left|\sigma_{k}\right|$ for all $k$. Then $\sigma_{1}=\operatorname{Re}\left(\sigma_{1}\right)$ is real and nonpositive, and there is a corresponding eigenvector containing only nonnegative entries. Moreover, $\operatorname{Re}\left(\sigma_{k}\right)<\sigma_{1} \leq 0$ for all $k$.

- If $c_{k 0} \neq 0$ for one or more $k$, then $\sigma_{1}<0$ and $A$ is invertible. All entries of $A^{-1}$ are nonpositive.

The proof is based on the Gerschgorin Circle Theorem and on the PerronFrobenius Theorem, see [2], page 55ff.

\subsubsection{Closed systems}

Suppose that there are no inflow reactions $R_{0 k}$ because $c_{0 k}=0$ for all $k$. If any of the degradation rates $c_{k 0}$ is nonzero, all molecules will disappear sooner or later and the system will tend to the trivial steady state $\lim _{t \rightarrow \infty} P(t, x)=$ $\delta_{\mathbf{0}}(x)$. Therefore, we only consider the more interesting case that $c_{k 0}=c_{0 k}=$ 0 for all $k$.

Proposition 5 Let $c_{k 0}=c_{0 k}=0$ for all $k$. Under the assumptions (A1) and (A2) the solution of the CME (8) with initial data $P(0, \cdot)=\delta_{\xi}(\cdot)$ for $\xi \in \mathbb{N}^{n}$ converges to a multinomial distribution:

$$
\lim _{t \rightarrow \infty} P(t, x)=\mathcal{M}(x,|\xi|, \bar{p}) .
$$

The vector $\bar{p} \in[0,1]^{n}$ is uniquely determined by the relations $A \bar{p}=\mathbf{0}$ and $|\bar{p}|=1$.

Proof. It follows from (6) that the sum over each column of $A$ is $\sum_{j=1}^{n} a_{j k}=$ 0 for each $k$. Hence, $\sigma_{1}=0$ is an eigenvalue with left eigenvector $(1, \ldots, 1)$. For $b=\mathbf{0}$ and constant $A$ the solutions of (16) are simply

$$
p^{(k)}(t)=\exp (t A) \varepsilon_{k}=T \exp (t J) T^{-1} \varepsilon_{k}, \quad \lambda(t) \equiv \mathbf{0}
$$


where $A=T J T^{-1}$ is the decomposition provided by the Jordan normal form (cf. Proposition 8 in the appendix). Thanks to the block structure of $J$ we can treat the matrix exponential of each Jordan block separately. Since the eigenvalue $\sigma_{1}$ is simple according to Proposition 4, the first Jordan block consists of only one entry, namely $\sigma_{1}=0$, and hence

$$
\exp \left(t J_{1}\right)=\exp \left(\sigma_{1} t\right)=1 .
$$

For any other Jordan block it can be shown that

$$
\lim _{t \rightarrow \infty} \exp \left(t J_{k}\right)=0
$$

(where "0" denotes the corresponding zero matrix) because $\operatorname{Re}\left(\sigma_{k}\right)<0$ for all $k>1$ by Proposition 4 . Hence, $\lim _{t \rightarrow \infty} \exp (t A)=T L T^{-1}$ with

$$
L=\lim _{t \rightarrow \infty} \exp (t J)=\left(\begin{array}{cccc}
1 & 0 & \cdots & 0 \\
0 & 0 & & \vdots \\
\vdots & & \ddots & 0 \\
0 & \cdots & 0 & 0
\end{array}\right)
$$

As a consequence, the parameter vectors $p^{(k)}(t)$ tend to well-defined limits and the system approaches an unique $^{2}$ steady state. The fact that $\exp (t A)$ converges implies that its derivative vanishes and thus

$$
0=\lim _{t \rightarrow \infty} \frac{d}{d t} \exp (t A)=\lim _{t \rightarrow \infty} \exp (t A) A=T L T^{-1} A .
$$

If $v^{(k)}$ is the $k$-th row of $T L T^{-1}$, then $v^{(k)} A=(0, \ldots, 0)$ which means that either $v^{(k)}=(0, \ldots, 0)$ or $v^{(k)}$ is a left eigenvector of $A$ to the eigenvalue $\sigma_{1}=0$. Since this eigenvalue is simple, the corresponding left eigenspace is one-dimensional and only consists of vectors of the form $r \cdot(1, \ldots, 1)$ with some $r \in \mathbb{R}$. This yields that

$$
T L T^{-1}=\left(\begin{array}{cccc}
r_{1} & r_{1} & \cdots & r_{1} \\
r_{2} & r_{2} & \cdots & r_{2} \\
\vdots & \vdots & & \vdots \\
r_{n} & r_{n} & \cdots & r_{n}
\end{array}\right)
$$

has $n$ identical columns and all parameter vectors $p^{(k)}(t)$ converge to the same limit:

$$
\lim _{t \rightarrow \infty} p^{(k)}(t)=\lim _{t \rightarrow \infty} \exp (t A) \varepsilon_{k}=T L T^{-1} \varepsilon_{k}=\left(\begin{array}{c}
r_{1} \\
\vdots \\
r_{n}
\end{array}\right)=: \bar{p}
$$

\footnotetext{
${ }^{2}$ Since the system always converges to this steady state it can never be in any other steady state.
} 
The limit $\bar{p}$ lies in the kernel of $A$ because $A \bar{p}=T J L T^{-1} \varepsilon_{k}=\mathbf{0}$. Moreover, it follows from (6) and $c_{k 0}=0$ that $|\bar{p}|=1$. We insert $\bar{p}$ into the solution distribution given in Theorem 1 and use that

$$
\mathcal{M}\left(\cdot, \xi_{1}, \bar{p}\right) \star \mathcal{M}\left(\cdot, \xi_{2}, \bar{p}\right)=\mathcal{M}\left(\cdot, \xi_{1}+\xi_{2}, \bar{p}\right)
$$

(see Lemma 2 in the appendix). This shows that the only steady state distribution is a multinomial distribution with parameters $\bar{p}$ and $|\xi|$.

Combining the above result with the representation (20), we obtain for a given initial distribution $\mu$ and every $x \in \mathbb{N}^{n}$

$$
\lim _{t \rightarrow \infty} P(t, x)=\sum_{\xi \in \mathbb{N}^{n}} \mu(\xi) \mathcal{M}(x,|\xi|, \bar{p})=\sum_{N=0}^{\infty} \mu_{N} \mathcal{M}(x, N, \bar{p}),
$$

where $\bar{p} \in[0,1]^{n}$ is uniquely determined by the relations $A \bar{p}=\mathbf{0}$ and $|\bar{p}|=1$, and $\mu_{N}$ is defined by

$$
\mu_{N}=\sum_{|\xi|=N} \mu(\xi)
$$

for $N \in \mathbb{N}$. Eq. (21) allows three interesting observations: (i) The steady steady is not unique and does depend on the initial distribution $\mu$, unless $c_{0 k}>0$ for some $k$. (ii) Fix some $N \in \mathbb{N}$. Then the limit distribution is unique and multinomial for the sub-class of initial distributions $\mu$ satisfying $\mu_{N}=1$. This sub-class particularly includes initial deterministic distributions and multinomial distributions $\mathcal{M}(\cdot, N, p)$, cf. Prop. 1. (iii) Closed system may evolve to Poissonian steady states (cf. Prop. 1). As an example, consider the initial distribution $\mu=\mathcal{P}(\cdot, \lambda)$ for some $\lambda \in[0, \infty)^{n}$. Then, $\mu$ can be interpreted as the joint propability distribution of $n$ independent Poission random variables $X_{1}, \ldots, X_{n}$. As a consequence, it is

$$
\begin{aligned}
& \mu_{N}=\mathbb{P}\left(X_{1}+\cdots+X_{n}=N\right) \\
& \mathbb{P}\left(X_{1}=x_{1} \ldots, X_{n}=x_{n} \mid X_{1}+\cdots+X_{n}=N\right)=\mathcal{M}(x, N, p)
\end{aligned}
$$

for $x \in \mathbb{N}^{n}$, see Remark 2 on page 10. Now, by Bayes formula, we obtain

$$
\lim _{t \rightarrow \infty} P(t, x)=\sum_{N=0}^{\infty} \mu_{N} \mathcal{M}(x, N, \bar{p})=\mathcal{P}(x, \bar{\lambda})
$$

with $\bar{\lambda}=|\lambda| \cdot \bar{p}_{i}$ for $i=1, \ldots, n$.

\subsubsection{Open systems}

Proposition 6 Let $c_{k 0} \neq 0$ for one or more $k$ and assume that (A1) and (A2) hold. Then, the solution of the CME (8) with initial data (9) converges to a Poisson distribution

$$
\lim _{t \rightarrow \infty} P(t, x)=\mathcal{P}(x, \bar{\lambda})
$$

where $\bar{\lambda}$ is the solution of $A \bar{\lambda}=-b$ and contains only nonnegative numbers. 
Remark. This agrees with a result proven in [8] which states that the stationary distribution of any species in an open first-order conversion network is a Poisson distribution.

Proof. By Proposition 4 the matrix $A$ is invertible and all eigenvalues have negative real part. The solutions of the differential equations (16) are

$$
p^{(k)}(t)=\exp (t A) \varepsilon_{k}, \quad \lambda(t)=(\exp (t A)-I) A^{-1} b
$$

and since $\lim _{t \rightarrow \infty} \exp (t A)=0$ (use the Jordan normal form as above) we obtain

$$
\lim _{t \rightarrow \infty} p^{(k)}(t)=\mathbf{0}, \quad \lim _{t \rightarrow \infty} \lambda(t)=-A^{-1} b=: \bar{\lambda} .
$$

All entries of $\bar{\lambda}$ are nonnegative because of Proposition 4. Inserting the limits into the solution distribution yields $\lim _{t \rightarrow 0} P(t, x)=\mathcal{P}(x, \bar{\lambda})$.

Combining the above result with the representation (20), we obtain for any given initial distribution $\mu$ and every $x \in \mathbb{N}^{n}$

$$
\lim _{t \rightarrow \infty} P(t, x)=\sum_{\xi \in \mathbb{N}^{n}} \mu(\xi) \mathcal{P}(x, \bar{\lambda})=\mathcal{P}(x, \bar{\lambda}),
$$

where $\bar{\lambda}$ is the solution of $A \bar{\lambda}=-b$ and contains only nonnegative numbers. Hence, for open systems the convergence and limit behavior is drastically different from that of closed systems. While in the latter case, the stationary distribution depends on the initial distribution, it is unique for open systems.

\section{Some simple examples}

In this subsection we illustrate our result by means of two low-dimensional examples that have been discussed by other authors.

\subsection{First example}

Consider one single substance $S_{1}$ and the two reaction channels

$$
\begin{array}{ll}
\text { Reaction } R_{01}: & \star \stackrel{c_{01}}{\longrightarrow} S_{1} \text { production from source or inflow } \\
\text { Reaction } R_{10}: & S_{1} \stackrel{c_{10}}{\longrightarrow} \star \text { degradation or outflow }
\end{array}
$$

with constant reaction rates $c_{01}>0$ and $c_{10}>0$. Suppose that at $t=0$ there are $\xi$ molecules $(\xi \in \mathbb{N})$ and let $P(t, x)$ be the probability that at time $t$ there are $x \in \mathbb{N}$ molecules of $S_{1}$. According to Theorem 1 this probability is given by

$$
P(t, x)=\sum_{k=0}^{\min \{\xi, x\}}\left(\begin{array}{l}
\xi \\
k
\end{array}\right) p^{k}(t)(1-p(t))^{\xi-k} \cdot \frac{\lambda^{x-k}(t)}{(x-k) !} e^{-\lambda(t)}
$$


where $p(t)=e^{-c_{10} t}$ and $\lambda(t)=c_{01}\left(1-e^{-t c_{10}}\right) / c_{10}$ are the solutions of the differential equations

$$
\begin{array}{ll}
\dot{p}(t)=-c_{10} p(t) & \dot{\lambda}(t)=-c_{10} \lambda(t)+c_{01} \\
p(0)=1 & \lambda(0)=0
\end{array}
$$

This result is stated in Todorovic [20, Sec. 8.4] or in Gardiner [9, Sec. 7.1]. Since $\lim _{t \rightarrow \infty} p(t)=0$ and $\lim _{t \rightarrow \infty} \lambda(t)=c_{01} / c_{10}$, the limit distribution

$$
\lim _{t \rightarrow \infty} P(t, x)=\frac{\bar{\lambda}^{x}}{x !} e^{-\bar{\lambda}}
$$

is the Poisson distribution with parameter $\bar{\lambda}=c_{01} / c_{10}$.

\subsection{Second example}

Now we consider the isomerization process with two species $S_{1}$ and $S_{2}$ and the two conversions

$$
\begin{array}{lll}
\text { Reaction } R_{12}: & S_{1} \stackrel{c_{12}}{\longrightarrow} S_{2} \\
\text { Reaction } R_{21}: & S_{2} \stackrel{c_{21}}{\longrightarrow} S_{1}
\end{array}
$$

with constant reaction rates $c_{12}>0$ and $c_{21}>0$. Suppose that at $t=0$ there are $\xi_{1}$ molecules of $S_{1}$ and $\xi_{2}$ molecules of $S_{2}$. Let $P(t, x)$ be the probability that at time $t \geq 0$ there are $x_{1}$ molecules of $S_{1}$ and $x_{2}$ molecules of $S_{2}$. Then, according to Theorem 1

$$
P(t, \cdot)=\mathcal{M}\left(\cdot, \xi_{1}, p^{(1)}(t)\right) \star \mathcal{M}\left(\cdot, \xi_{2}, p^{(2)}(t)\right) .
$$

with parameter vectors $p^{(1)}(t)$ and $p^{(2)}(t)$ given by

$$
p^{(1)}(t)=\exp (t A) \varepsilon_{1}, \quad p^{(2)}(t)=\exp (t A) \varepsilon_{2}, \quad A=\left(\begin{array}{rr}
-c_{12} & c_{21} \\
c_{12} & -c_{21}
\end{array}\right) .
$$

The above formulas can be simplified if the diagonalisation $A=T D T^{-1}$ with

$$
T=\left(\begin{array}{cc}
1 & c_{21} \\
-1 & c_{12}
\end{array}\right), \quad D=\left(\begin{array}{cc}
-d & 0 \\
0 & 0
\end{array}\right), \quad T^{-1}=\frac{1}{d}\left(\begin{array}{cc}
c_{12} & -c_{21} \\
1 & 1
\end{array}\right), \quad d=c_{12}+c_{21}
$$

is used to compute the matrix exponential $\exp (t A)=T \exp (t D) T^{-1}$. This yields

$$
p^{(1)}(t)=\frac{1}{d}\left(\begin{array}{c}
e^{-t d} c_{12}+c_{21} \\
\left(1-e^{-t d}\right) c_{12}
\end{array}\right), \quad p^{(2)}(t)=\frac{1}{d}\left(\begin{array}{c}
\left(1-e^{-t d}\right) c_{21} \\
e^{-t d} c_{21}+c_{12}
\end{array}\right) .
$$

Since $\left|p^{(1)}(t)\right|=\left|p^{(2)}(t)\right|=1$ we find that

$$
\left(1-\left|p^{(k)}\right|\right)^{\xi_{k}-|x|}=0^{\xi_{k}-|x|}=\delta_{\xi_{k}}(|x|) .
$$


As a consequence,

$$
\begin{aligned}
\mathcal{M}\left(x, \xi_{k}, p^{(k)}\right) & =\frac{\xi_{k} !}{x_{1} ! x_{2} !(1-|x|) !}\left(p_{1}^{(k)}\right)^{x_{1}}\left(p_{2}^{(k)}\right)^{x_{2}}\left(1-\left|p^{(k)}\right|\right)^{\xi_{k}-|x|} \\
& =\delta_{\xi_{k}}(|x|) \frac{\xi_{k} !}{x_{1} !\left(1-x_{1}\right) !}\left(p_{1}^{(k)}\right)^{x_{1}}\left(1-p_{1}^{(k)}\right)^{\xi_{k}-x_{1}} \\
& =\delta_{\xi_{k}}(|x|) \mathcal{B}\left(x_{1}, \xi_{k}, p_{1}^{(k)}\right)
\end{aligned}
$$

for $k=1$ or $k=2$, where $\mathcal{B}\left(\cdot, \xi_{k}, p_{1}^{(k)}\right)$ denotes the binomial distribution. This means that the total number of particles must be constant, which is reasonable for the above system. The two-dimensional convolution of two multinomial distribution now reduces to a one-dimensional convolution of two binomial distributions:

$$
\begin{aligned}
P(t, x) & =\sum_{z} \delta_{\xi_{k}}(|z|) \mathcal{B}\left(z_{1}, \xi_{1}, p_{1}^{(1)}\right) \delta_{\xi_{2}}(|x-z|) \mathcal{B}\left(x_{1}-z_{1}, \xi_{2}, p_{1}^{(2)}\right) \\
& =\delta_{|\xi|}(|x|) \sum_{z_{1}} \mathcal{B}\left(z_{1}, \xi_{1}, p_{1}^{(1)}\right) \mathcal{B}\left(x_{1}-z_{1}, \xi_{2}, p_{1}^{(2)}\right) .
\end{aligned}
$$

Since both $p_{1}^{(1)}(t)$ and $p_{1}^{(2)}(t)$ converge to the same value $\bar{p}=c_{21} /\left(c_{21}+c_{12}\right)$ the stationary distribution is

$$
\lim _{t \rightarrow \infty} P(t, \cdot)=\delta_{|\xi|}(|x|)\left(\mathcal{B}\left(\cdot, \xi_{1}, \bar{p}\right) * \mathcal{B}\left(\cdot, \xi_{2}, \bar{p}\right)\right)=\delta_{|\xi|}(x) \mathcal{B}(\cdot, \xi, \bar{p})
$$

because the convolution of two binomials with the same parameter is again a binomial (cf. [14]). In [13] the same stationary distribution was obtained in a different way.

\section{Outlook}

In this article we derive an explicit formula for the exact solution of the CME under the assumption that only monomolecular reactions occur in the underlying reaction system. For the generic case of deterministic initial conditions, the solution turns out to be the convolution of multinomial and product Poisson distributions with time-dependent parameters evolving according to the traditional reaction-rate equations. To our knowlegde this is the first report of an general solution for arbitrary initial conditions. All previously derived results on monomolecular reaction systems can be restated in terms of our representation highlighting the structure of the system, as has been shown in Sec. 5. Exploiting the structure of the solution, many interesting features of the time-dependent distribution have been expressed in terms of the timedepending parameters specifying the multinomial and the product Poisson distribution.

Our results presented so far are applicable to reaction systems including conversion, production and degradation reactions, as specified in (1). The 
question arises whether Theorem 1 can be extended to a larger class of systems including, e.g.,

$$
\begin{array}{ll}
S_{j} \stackrel{c}{\longrightarrow} S_{k}+S_{l} & \text { splitting reaction } \quad(j \neq k, l) \\
S_{j} \stackrel{c}{\longrightarrow} S_{j}+S_{k} & \text { catalytic production } \quad(j \neq k) \\
S_{j} \stackrel{c}{\longrightarrow} S_{j}+S_{j} & \text { autocatalytic production. }
\end{array}
$$

As a first step in this direction, we discuss the case of one single species evolving according to an autocatalytic reaction with nonnegative rate $c(t)$. Theorem 1 cannot be applied to this situation because (22) is not a monomolecular reaction in the sense of Section 2. Nevertheless, we can state an explicit formula for the probability distribution that solves the corresponding CME.

Proposition 7 Suppose that at $t=0$ there are $\xi \in \mathbb{N} \backslash\{0\}$ molecules of a species $S$ taking part in the autocatalytic reaction

$$
S \stackrel{c}{\longrightarrow} S+S
$$

with rate $c(t) \geq 0$. If $P(t, m)$ denotes the probability to find $m \in \mathbb{N}$ molecules of $S$ at time $t$, then

$$
P(t, m)=\left\{\begin{array}{cc}
\left(\begin{array}{c}
m-1 \\
m-\xi
\end{array}\right) p^{\xi}(t)(1-p(t))^{m-\xi} & \text { for } m \geq \xi \\
0 & \text { for } m<\xi
\end{array}\right.
$$

with parameter $p(t)$ obtained by solving the differential equation $\dot{p}(t)=-c(t) p(t)$ with $p(0)=1$.

Proof. It has to be shown that (23) solves the CME

$$
\frac{\partial}{\partial t} P(t, m)=c(t)(m-1) P(t, m-1)-c(t) m P(t, m)
$$

with initial data $P(0, m)=\delta_{\xi}(m)$. Taking the derivative of (23) and omitting the argument " $(t)$ " gives

$$
\begin{aligned}
\frac{\partial}{\partial t} P(t, m) & =\left(\begin{array}{l}
m-1 \\
m-\xi
\end{array}\right)\left(\xi p^{\xi-1}(1-p)^{m-\xi}-p^{\xi}(m-\xi)(1-p)^{m-\xi-1}\right) \dot{p}(t) \\
& =\left(\frac{\xi}{p}-\frac{m-\xi}{1-p}\right) \dot{p} P(t, m) \\
& =\left(-c \xi+c p \frac{m-\xi}{1-p}\right) P(t, m) \\
& =-c m P(t, m)+c \frac{m-\xi}{1-p} P(t, m) \\
& =-c m P(t, m)+c(m-1) P(t, m-1) .
\end{aligned}
$$

for all $m \geq \xi$, where we have used that $1-p(t)>0$ and $p(t)>0$ for $t>0$. At $t=0$ we obtain

$$
\left(\begin{array}{c}
m-1 \\
m-\xi
\end{array}\right) p^{\xi}(0)(1-p(0))^{m-\xi}=\left(\begin{array}{c}
m-1 \\
m-\xi
\end{array}\right) 1^{\xi} \delta_{\xi}(m)=\delta_{\xi}(m)=P(0, m) .
$$


This proves that (23) is indeed the solution of (24).

Remark. The distribution (23) is a shifted variant of the negative binomial distribution $\tilde{\mathcal{B}}$ defined by

$$
\tilde{\mathcal{B}}(m, r, q)=\left(\begin{array}{c}
r+m-1 \\
m
\end{array}\right) p^{r}(1-p)^{m} .
$$

for all $p \in[0,1]$ and $r>0$, see [14]. It is easy to see that $P(t, m)=\tilde{\mathcal{B}}(m-$ $\xi, \xi, p)$. Since it is known that

$$
\mathbb{E}(\tilde{\mathcal{B}}(\cdot, r, q))=\sum_{m=0}^{\infty} m \tilde{\mathcal{B}}(m, r, q)=\frac{q r}{1-q}
$$

the expectation of $P(t, \cdot)$ is

$$
\begin{aligned}
\mathbb{E}(P(t, \cdot)) & =\sum_{m=0}^{\infty}(m-\xi+\xi) \tilde{\mathcal{B}}(m-\xi, \xi, 1-p(t)) \\
& =\mathbb{E}(\tilde{\mathcal{B}}(\cdot, \xi, 1-p(t)))+\xi \\
& =\frac{(1-p(t)) \xi}{p(t)}+\xi=\frac{\xi}{p(t)}=\xi e^{c t}
\end{aligned}
$$

which is again the solution of the "classical" rate equation $\dot{y}(t)=c y(t)$ with initial value $y(0)=\xi$.

Proposition 7 provides an analytic solution for isolated autocatalytic reactions, but unfortunately we have not yet found a way to treat systems where autocatalytic reactions occur together with and production, conversion or degradation reactions. For example, the joint probability distribution of the system

$$
\begin{aligned}
& * \stackrel{c_{1}}{\longrightarrow} S \\
& S \stackrel{c_{2}}{\longrightarrow} * \\
& S \stackrel{c_{3}}{\longrightarrow} S+S
\end{aligned}
$$

cannot be derived from the results of this paper. We only know that somehow this distribution must "interpolate" between the Poisson, binomial and negative binomial distributions, because each of these is obtained in a special case. We believe, however, that Proposition 7 and Theorem 1 can be combined and extended in such a way that analytic solutions of the CME are obtained even when both monomolecular and autocatalytic reactions are possible. Work in this direction is in progress.

Acknowledgement. This work was supported by the DFG Research Center MATHEON "Mathematics for key technologies: Modelling, simulation, and optimization of real-world processes", Berlin. 


\section{References}

1. A. Alfonsi, E. Cancès, G. Turinici, B. D. Ventura, and W. Huisinga. Adaptive simulation of hybrid stochastic and deterministic models for biochemical systems. ESAIM Proceeding, 14:1-13, 2005.

2. D. H. Anderson. Compartmental Modeling and Tracer Kinetics. Number 50 in Lecture Notes in Biomathematics. Springer, Berlin, 1983.

3. K. Burrage and T. Tian. Poisson Runge-Kutta methods for chemical reaction systems. In Proceedings of the Hong Kong Conference on Scientific Computing, 2003 (to appear).

4. K. Burrage, T. Tian, and P. Burrage. A multi-scaled approach for simulating chemical reaction systems. Progress in Biophysics and Molecular Biology 85:217-234, 2004.

5. Y. Cao, D. Gillespie, and L. Petzold. The slow-scale stochastic simulation algorithm. J. Chem. Phys., 122(1):014116, 2005.

6. I. Darvey and P. Staff. Stochastic approach to first-order chemical reaction kinetics. J. Chem. Phys., 44(3):990-997, 1966.

7. C. P. Fall, E. S. Marland, J. M. Wagner, and J. J. Tyson. Computational Cell Biology, volume 20 of Interdisciplinary Applied Mathematics. Springer, New York, 2002.

8. C. Gadgil, C.-H. Lee, and H. G. Othmer. A stochastic analysis of first-order reaction networks. Bull. Math. Biol., 67:901-946, 2005.

9. C. W. Gardiner. Handbook of Stochastic Methods. Springer, Berlin, 2nd enlarged edition edition, 1985.

10. M. A. Gibson and J. Bruck. Efficient exact stochastic simulation of chemical systems with many species and many channels. J. Phys. Chem. A, 104:18761889, 2000.

11. D. T. Gillespie. A general method for numerically simulating the stochastic time evolution of coupled chemical reactions. J. Comput. Phys., 22:403-434, 1976.

12. D. T. Gillespie. Approximate accelerated stochastic simulation of chemically reacting systems. Journal of Chemical Physics, 115(4):1716-1733, 2001.

13. D. T. Gillespie. The chemical Langevin and Fokker-Planck equations for the reversible isomerization reaction. J. Phys. Chem., 106:5063-5071, 2002.

14. M. A. Golberg. An Introduction to Probability Theory with Statistical Applications. Plenum Press, New York and London, 1984.

15. E. L. Haseltine and J. B. Rawlings. Approximate simulation of coupled fast and slow reactions for stochastic chemical kinetics. Journal of Chemical Physics, 117(15):6959-6969, 2002.

16. A. Iserles, H. Z. Munthe-Kaas, S. P. Nørsett, and A. Zanna. Lie-group methods. Acta Numerica, 9:215-365, 2000.

17. S. Kotz, N. L. Johnson, and C. B. Read, editors. Encyclopedia of statistical sciences, volume 5. Wiley, New York, Chichester, Brisbane, Toronto, Singapore, 1985.

18. C. V. Rao and A. P. Arkin. Stochastic chemical kinetics and the quasi-steadystate assumption: Application to the Gillespie algorithm. J. Chem. Phys., 118(11):4999-5010, 2003.

19. H. Salis and Y. Kaznessis. Accurate hybrid simulation of a system of coupled chemical or biochemical reactions. J. Chem. Phys., 122, 2005.

20. P. Todorovic. An introduction to stochastic processes and their applications. Springer, New York, 1992.

\section{A Appendix}

Lemma 1 For all $p \in[0,1]^{n}$ and $x \in \mathbb{N}^{n}$ with $|x| \leq N \in \mathbb{N}$ the multinomial distribution $\mathcal{M}(x, N, p)$ can be represented as a product of binomial distributions

$$
\mathcal{M}(x, N, p)=\prod_{k=1}^{n} \mathcal{B}\left(x_{k}, s_{k}, r_{k}\right)
$$


with

$$
\begin{aligned}
\mathcal{B}\left(x_{k}, s_{k}, r_{k}\right) & =\left(\begin{array}{c}
s_{k} \\
x_{k}
\end{array}\right) r_{k}^{x_{k}}\left(1-r_{k}\right)^{s_{k}-x_{k}}, \\
s_{k} & =N-\sum_{j=1}^{k-1} x_{j}, \quad r_{k}=\frac{p_{k}}{1-\sum_{j=1}^{k-1} p_{j}} .
\end{aligned}
$$

Proof by induction. For $n=1$ the binomial and the multinomial distributions coincide and the assertion is trivial. If (25) holds for $n-1$ we have

$$
\begin{aligned}
\prod_{k=1}^{n} \mathcal{B}\left(x_{k}, s_{k}, r_{k}\right) & =\mathcal{M}(\tilde{x}, N, \tilde{p}) \mathcal{B}\left(x_{n}, s_{n}, r_{n}\right) \\
& =N ! \frac{(1-|\tilde{p}|)^{N-|\tilde{x}|}}{(N-|\tilde{x}|) !} \prod_{k=1}^{n-1} \frac{p_{k}^{x_{k}}}{x_{k} !} \cdot \frac{s_{n} !}{x_{n} !\left(s_{n}-x_{n}\right) !} r_{n}^{x_{n}}\left(1-r_{n}\right)^{s_{n}-x_{n}}
\end{aligned}
$$

with $\tilde{x}=\left(x_{1}, \ldots, x_{n-1}\right)^{T}$ and $\tilde{p}=\left(p_{1}, \ldots, p_{n-1}\right)^{T}$. Since

$$
r_{n}=\frac{p_{n}}{1-|\tilde{p}|}, \quad 1-r_{n}=\frac{1-|p|}{1-|\tilde{p}|}, \quad s_{n}=N-|\tilde{x}|
$$

by definition, we obtain

$$
\begin{aligned}
(1-|\tilde{p}|)^{N-|\tilde{x}|} r_{n}^{x_{n}}\left(1-r_{n}\right)^{s_{n}-x_{n}} & =(1-|\tilde{p}|)^{N-|\tilde{x}|}\left(\frac{p_{n}}{1-|\tilde{p}|}\right)^{x_{n}}\left(\frac{1-|p|}{1-|\tilde{p}|}\right)^{s_{n}-x_{n}} \\
& =(1-|\tilde{p}|)^{N-|\tilde{x}|-s_{n}} p_{n}^{x_{n}}(1-|p|)^{s_{n}-x_{n}} \\
& =p_{n}^{x_{n}}(1-|p|)^{N-|x|}
\end{aligned}
$$

Moreover, the relations $s_{n}-x_{n}=N-|x|$ and $s_{n}=N-|\tilde{x}|$ yield

$$
\frac{1}{(N-|\tilde{x}|) !} \cdot \frac{s_{n} !}{x_{n} !\left(s_{n}-x_{n}\right) !}=\frac{1}{x_{n} !(N-|x|) !} .
$$

Inserting (27) and (28) into (26) gives

$$
\prod_{k=1}^{n} \mathcal{B}\left(x_{k}, s_{k}, r_{k}\right)=N ! \prod_{k=1}^{n-1} \frac{p_{k}^{x_{k}}}{x_{k} !} \cdot \frac{p_{n}^{x_{n}}(1-|p|)^{N-|x|}}{x_{n} !(N-|x|) !}=\mathcal{M}(x, N, p) .
$$

Lemma 2 For all $p \in[0,1]^{n}$ and $N_{1}, N_{2} \in \mathbb{N}^{n}$

$$
\mathcal{M}\left(\cdot, N_{1}, p\right) * \mathcal{M}\left(\cdot, N_{2}, p\right)=\mathcal{M}\left(\cdot, N_{1}+N_{2}, p\right)
$$

Proof. For $n=1$ the multinomial distributions reduce to binomial distributions and the convolution reads

$$
\mathcal{B}\left(\cdot, N_{1}, p\right) * \mathcal{B}\left(\cdot, N_{2}, p\right)=\mathcal{B}\left(\cdot, N_{1}+N_{2}, p\right)
$$

This equation is proven in most textbooks on probability theory, e.g. [14]. For $n>1$ we can use Lemma 1 with

$$
s_{k}^{(1)}=N_{1}-\sum_{j=1}^{k-1} z_{j}, \quad s_{k}^{(2)}=N_{2}-\sum_{j=1}^{k-1}\left(x_{j}-z_{j}\right), \quad r_{k}=\frac{p_{n}}{1-\sum_{j=1}^{k-1} p_{j}} .
$$


and obtain via (29) that

$$
\begin{aligned}
& \sum_{z_{n}} M\left(z, N_{1}, p\right) \mathcal{M}\left(x-z, N_{2}, p\right) \\
= & \sum_{z_{n}} \prod_{k=1}^{n} \mathcal{B}\left(z_{k}, s_{k}^{(1)}, r_{k}\right) \mathcal{B}\left(x_{k}-z_{k}, s_{k}^{(2)}, r_{k}\right) \\
= & \left(\prod_{k=1}^{n-1} \mathcal{B}\left(z_{k}, s_{k}^{(1)}, r_{k}\right) \mathcal{B}\left(x_{k}-z_{k}, s_{k}^{(2)}, r_{k}\right)\right) \sum_{z_{n}} \mathcal{B}\left(z_{n}, s_{n}^{(1)}, r_{n}\right) \mathcal{B}\left(x_{n}-z_{n}, s_{n}^{(2)}, r_{n}\right) \\
= & \left(\prod_{k=1}^{n-1} \mathcal{B}\left(z_{k}, s_{k}^{(1)}, r_{k}\right) \mathcal{B}\left(x_{k}-z_{k}, s_{k}^{(2)}, r_{k}\right)\right) \cdot \mathcal{B}\left(x_{n}, s_{n}, r_{n}\right) .
\end{aligned}
$$

with $s_{n}=s_{n}^{(1)}+s_{n}^{(2)}=N_{1}+N_{2}-\sum_{j=1}^{k-1} x_{j}$. By recursion this yields

$$
\begin{aligned}
\left(\mathcal{M}\left(\cdot, N_{1}, p\right) * \mathcal{M}\left(\cdot, N_{2}, p\right)\right)(x) & =\sum_{z_{1}} \sum_{z_{2}} \cdots \sum_{z_{n}} M\left(z, N_{1}, p\right) \mathcal{M}\left(x-z, N_{2}, p\right) \\
& =\prod_{i=1}^{n} \mathcal{B}\left(x_{i}, s_{i}, r_{i}\right)=\mathcal{M}(x, N, p)
\end{aligned}
$$

Proposition 8 (Jordan normal form) For any arbitrary matrix $A \in \mathbb{C}^{n \times n}$ there is a decomposition $T^{-1} A T=J$ with an invertible matrix $T \in \mathbb{C}^{n \times n}$ and

$$
J=\left(\begin{array}{cccc}
J_{1} & 0 & \cdots & 0 \\
0 & J_{2} & & \vdots \\
\vdots & & \ddots & 0 \\
0 & \cdots & 0 & J_{\kappa}
\end{array}\right), \quad J_{k}=\left(\begin{array}{ccccc}
\lambda_{k} & 1 & 0 & \cdots & 0 \\
0 & \lambda_{k} & 1 & \ddots & \vdots \\
\vdots & & \ddots & \ddots & 0 \\
\vdots & & & \lambda_{k} & 1 \\
0 & \cdots & \cdots & 0 & \lambda_{k}
\end{array}\right) \in \mathbb{C}^{n_{k} \times n_{k}}
$$

with $n_{1}+n_{2}+\ldots+n_{\kappa}=n$. Each $\lambda_{k}$ is an eigenvalue of $A$, but there can be several Jordan blocks $J_{k}$ belonging to the same eigenvalue.

Proof: See any textbook on linear algebra. 